\title{
Domain-specific conflict adaptation without feature repetitions
}

\author{
Çağlar Akçay • Eliot Hazeltine
}

Published online: 15 March 2011

(C) Psychonomic Society, Inc. 2011

\begin{abstract}
An influential account of how cognitive control deals with conflicting sources of information holds that conflict is monitored by a module that automatically recruits attention to resolve the conflict. This leads to reduced effects of conflict on the subsequent trial, a phenomenon termed conflict adaptation. A prominent question is whether control processes are domain specific-that is, recruited only by the particular type of conflict they resolve. Previous studies that have examined this question used two-choice tasks in which feature repetition effects could be responsible for domain-specific adaptation effects. We report two experiments using four-choice (Experiment 1) and five-choice (Experiment 2) tasks that contain two types of irrelevant sources of potentially conflicting information: stimulus location (Simon conflict) and distractors (flanker conflict). In both experiments, we found within-type conflict adaptation for both types of conflict after eliminating trials on which stimulus features were repeated from one trial to the next. Across-type conflict adaptation, however, was not significant. Thus, conflict adaptation was due to domain-specific recruitment of cognitive control. Our results add converging evidence to the idea that multiple independent control processes are involved in reactive cognitive control, although whether control is always local remains to be determined.
\end{abstract}

Ç. Akçay $(\bowtie)$

Department of Psychology, University of Washington,

Box 351525, Seattle, WA 98195, USA

e-mail: caglar@u.washington.edu

E. Hazeltine

Department of Psychology, University of Iowa,

Iowa City, IA 52242, USA
Keywords Attention and executive control - Repetition effects · Decision making · Cognitive and attentional control

The cognitive system is confronted with multiple sources of information from the environment, some of which may suggest conflicting responses. For instance, the visual environment usually contains multiple stimuli, most of them irrelevant to the action at hand and some presenting competing courses of action. One might think of a basketball player choosing to pass the ball to a teammate, as opposed to an opponent or a spectator. How the cognitive system achieves the regulation of information in the planning of actions is a problem that has been extensively studied (e.g., Eriksen \& Eriksen, 1974; MacLeod, 1991; Simon \& Rudell, 1967).

The general finding from the simple laboratory tasks is that response selection is hampered (i.e., slower or less accurate) when irrelevant but conflicting information is presented to participants. For instance, in a task in which a red color calls for a right-sided press response, participants perform the response more quickly if the stimulus is presented in the right visual field (a corresponding trial) rather than in the left (a noncorresponding trial). However, this effect decreases in magnitude or even reverses on trials immediately following noncorresponding trials. The latter phenomenon, called conflict adaptation (Gratton, Coles, \& Donchin, 1992; Kerns, Cohen, MacDonald, Cho, Stenger, \& Carter, 2004; Kunde, 2003; Stürmer, Leuthold, Soetens, Schröter, \& Sommer, 2002), prompted an influential account of cognitive control called the conflict-monitoring hypothesis.

Under the conflict-monitoring hypothesis, control is modulated by a conflict-monitoring process that registers competition among coactive response options whenever 
conflicting information is present in the system (Botvinick, Braver, Barch, Carter, \& Cohen, 2001). The conflict monitor feeds into an attentional system that affects the activation of the responses by attenuating their inputs, so that a feedback loop is formed. One attractive feature of this model is that adjustment of the attentional system is automatic; that is, it does not require any intervention from additional control processes once the task representations are set up, avoiding the "homunculus problem" (Monsell \& Driver, 2000). Since it was first proposed, there have been alternative accounts of conflict monitoring to explain conflict adaptation in terms of repetitions of stimulus and response features (Hommel, Proctor, \& Vu, 2004; Mayr, Awh, \& Laurey, 2003). However, recent studies have established that both repetition effects and control contribute to conflict adaptation (Akçay \& Hazeltine, 2007; Notebaert \& Verguts, 2007).

An automatic feedback loop through conflict monitoring is a plausible process in tasks that involve one type of conflicting information only. But what happens in situations in which multiple sources of information, some helpful and some misleading, converge upon the system simultaneously? Is the control recruited by conflict monitoring global so that it affects all ongoing processes, or is it local so that it affects only a subset that are relevant to the conflict?

Determining the locus of control involves two related but distinct questions: Is control local with respect to the type of conflict that recruited it, and is control local with respect to the task in which it was first recruited (Egner, 2008)? We call the former question the domain specificity and the latter the task specificity of control.

Both task specificity and domain specificity have received much recent attention (e.g., Akçay \& Hazeltine, 2008; Egner, Delano, \& Hirsch, 2007; Kiesel, Kunde, \& Hoffman, 2006; Notebaert \& Verguts, 2008). However, in a recent review, Egner (2008) rightly argued that many of these studies did not distinguish between the questions of task and domain specificity, since their designs included two tasks with different types of conflict. In one exception to this, we found that conflict adaptation was task specific even within a conflict type (in this case, conflict from irrelevant stimulus location-i.e., Simon conflict; Akçay \& Hazeltine, 2008). In the present study, we examined whether conflict adaptation is domain specific within a task. That is, does conflicting information of one type (such as irrelevant visual distractors-i.e. flanker conflict) lead to conflict adaptation in a different type of conflict (such as Simon conflict) within a single task?

A few studies have examined the question of domain specificity by factorially combining different types of conflict within a single task. For example, Egner et al. (2007) combined Stroop-type conflict with Simon conflict within a single task. They observed that Simon conflict led to a decrease only in the Simon effect, whereas Stroop conflict led to a decrease only in the Stroop effects. In another study, Wendt, Kluwe, and Peters (2006) factorially crossed Simon conflict with conflict from distractor stimuli (flanker conflict). Critically, they found that Simon and flanker effects interacted underadditively, suggesting that these two types of conflict may not be completely independent (see also Hommel, 1997). Nevertheless, conflict adaptation was still seen only within a task. Funes and colleagues (Funes, Lupiáñez, \& Humpreys, 2010) used a single task that combined Simon and flanker conflict and found that conflict adaptation was local with respect to the conflict type. However, the authors did not factorially cross conflict types, which, in practice, resulted in markedly different displays for the two types of conflict (e.g., target always presented at the center for flanker conflict trials and always laterally on the Simon conflict trials), which may have generated an implicit task differentiation (Akçay \& Hazeltine, 2008).

A significant problem for all of these studies is that they used two-choice tasks and, therefore, were not able to distinguish between repetition effects and conflict adaptation directly (Hommel et al., 2004; Mayr et al., 2003). In fact, Wendt et al. (2006) concluded that within-domain conflict adaptation was due mostly to the repetition of stimulus-response combinations. This argument has good explanatory power in dealing with domain-specific conflict adaptation: Given that repetition effects, by definition, act on repeated stimulus features (e.g., repeated flankers), from the perspective of repetition effects, only local domainspecific effects are expected.

In sum, when there are only two relevant feature values for each stimulus dimension, the repetition effect account makes the same prediction as a local conflict-monitoring account. However, when four (or more) possible feature values are used, we can identify the locus of conflict adaptation as either global or local, and if local, we can then identify whether it is due solely to feature repetitions or domain-specific control.

\section{Experiment 1}

To identify the locus of conflict adaptation, we conducted an experiment in which we factorially crossed flanker and Simon conflict within a common task. In our task, a target (a colored ring) was embedded among two irrelevant distractors (themselves colored rings). The relevant feature determining the response was color. The target could appear in one of four locations (see Fig. 1), and this location could be corresponding or noncorresponding with respect to the response location (Simon conflict) and distractor color could be congruent or incongruent with response to the 


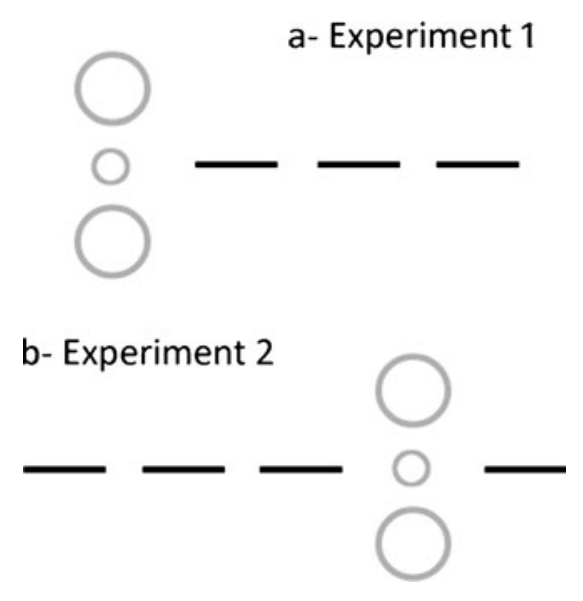

Fig. 1 Example displays representing the stimuli used in a Experiment 1 and $\mathbf{b}$ Experiment 2. The background was actually black, circles were colored (see the text for RGB values), and placeholders were white

target-color/response (flanker conflict). Each trial, therefore, involved any combination of flanker and Simon conflicts. Furthermore, we used a four-choice task and designed trial sequences so that stimulus (and response) features never were repeated (with one exception, addressed below). Thus, we can safely attribute any conflict adaptation to cognitive control. If conflict adaptation is domain specific, we should see a decrease in the Simon effect only after Simon conflict and not after flanker conflict. Similarly, the flanker effect should be reduced only after flanker conflict, and not after Simon conflict. On the other hand, if we see a decrease in the Simon effect after flanker conflict and/or a decrease in the flanker effect after Simon conflict, this would be evidence against the domain specificity of control recruited by that type of conflict.

\section{Method}

Participants Eighteen undergraduates participated in the experiment in exchange for course credit. All had normal or corrected-to-normal vision and were right-handed.

Apparatus and stimuli The stimuli were presented on a 17-in. LCD monitor controlled by an IBM-compatible PC from a distance of approximately $50 \mathrm{~cm}$. The stimuli were colored rings. Each stimulus display included three colored rings, two of which were distractors and the remaining one the target (see Fig. 1a). The distractors were larger than the target, subtending approximately $2.6^{\circ}$ of visual angle, whereas targets subtended $1.8^{\circ}$. The target was displayed in one of four locations in the center of the screen, so that the distance between locations was $4.0^{\circ}$. The distractors were presented directly above and below the target $2.5^{\circ}$ apart, center to center. We used red, green, blue, and yellow (RGB values: $237-27-36,37-177-77,0-163-232$, and 255255-0, respectively) as stimulus colors, and they mapped to the responses in this arrangement from left to right. Response keys were the keys $\mathrm{h}-\mathrm{j}-\mathrm{k}-\mathrm{l}$ on a standard keyboard, and participants used their index, middle, ring, and pinky fingers of their right hands.

Design and procedure Participants completed 20 blocks of 65 trials. We generated pseudorandom stimulus lists that satisfied the following criteria: (1) One quarter of the trials (excepting the first trial of each block) were corresponding and congruent, one quarter were corresponding and incongruent, one quarter were noncorresponding and congruent, and one quarter were noncorresponding and incongruent; (2) there was no repetition of response (target color) on two consecutive trials; (3) there was no repetition of target location on two consecutive trials; and (4) there was no repetition of distractor color on two consecutive trials. We adopted these constraints in order to collect enough trials per cell that are nonrepetitions of features for a four-way ANOVA. The design leaves the possibility of trial contingencies where a distractor on a given trial corresponds to the location on the following trial and vice versa, as well as trials where the preceding location (or distractor) corresponds to the response on the current trial or vice versa. These trials are termed negative priming trials and may mask conflict adaptation effects (Bugg, 2008). Therefore, in all the analyses reported below, we excluded these trials.

Each trial began with a 500-ms foreperiod with only a black background visible. Next, the stimulus display consisting of four colored rings appeared. The display stayed on for $150 \mathrm{~ms}$, which was followed by a response interval of $1,850 \mathrm{~ms}$, during which only the background was visible again. Participants were instructed to respond as quickly and accurately as possible. The next trial began immediately after the response interval if the response was correct. If the participant did not respond or responded incorrectly, the response interval was followed by a 2 -s black screen, and a tone was played for $500 \mathrm{~ms}$ to give feedback.

\section{Results}

The first block was considered practice and was not included in the analyses. We also excluded the first trial of each block. Trials with incorrect responses (5.9\%), trials with responses faster than $200 \mathrm{~ms}(2.0 \%)$, and trials with responses slower than $1,500 \mathrm{~ms}(2.0 \%)$, as well as trials following these, were excluded from response time (RT) analyses (a total of $16 \%$ of the trials). We submitted the RTs and error rates to a $2 \times 2 \times 2 \times 2$ ANOVA with factors Simon correspondence, flanker congruity, Simon correspondence on previous trial, and flanker congruity on previous trial. With this approach, each cell, on average, 
contained 44 trials. Error rates followed the pattern in RTs closely; for brevity, we discuss only the results for RT. The ANOVA statistics are reported in Table 1. We also report effect sizes (Cohen's $f$ ) calculated for each effect in Table 1. Cohen's $f$ was calculated from partial $\eta^{2}$, using the software G*Power 3.1.2 (http://www.psycho.uni-duesseldorf.de/ abteilungen/aap/gpower3/; Faul, Erdfelder, Lang, \& Buchner, 2007).

All main effects except flanker correspondence on previous trial were significant (see Table 1 for the statistical tests and effect sizes). Trials were $33 \mathrm{~ms}$ faster when the previous Simon correspondence was corresponding versus noncorresponding, $79 \mathrm{~ms}$ faster when Simon correspondence was corresponding versus noncorresponding, and $43 \mathrm{~ms}$ faster when flankers were congruent versus incongruent.

The conflict adaptation effects are indicated by a two-way interaction between correspondence on the previous trial and the that on the current trial. There was a significant two-way interaction between previous Simon conflict and current Simon conflict, indicating significant conflict adaptation within Simon conflict. Simon effect decreased from $93 \mathrm{~ms}$ after corresponding trials to $64 \mathrm{~ms}$ after noncorresponding trials (see Fig. 2a). The two-way interaction between previous flanker conflict and current flanker conflict was also significant. The flanker effect decreased from $51 \mathrm{~ms}$ after flanker congruent trials to $35 \mathrm{~ms}$ after flanker incongruent trials (see Fig. 2b). Thus, within-type conflict adaptation was significant in both Simon and flanker conflicts.

There was, however, no evidence for across-type conflict adaptation. Simon correspondence on the previous trial did not interact significantly with flanker congruity on the current trial (see Fig. 3a), and flanker conflict on the previous trial did not interact with Simon conflict on the current trial (see Fig. 3b).

No other two-way interactions and none of the three- and four-way interactions were significant at $p=.05$ or had large effect sizes (Table 1).

\section{Experiment 2}

In Experiment 1, we used pseudorandom lists that restricted the stimuli to be nonrepetitions within each feature. This introduced some unwanted contingencies in the stimulus lists, particularly after noncorresponding/incongruent trials, after which there were fewer possible alternatives than after corresponding/congruent trials. This may have affected performance in such a way as to lead to local control effects. To rule out this possibility, we conducted a second experiment in which the stimuli were selected at random, with the only constraints that the response was not repeated on consecutive trials and that Simon and flanker conflicts were orthogonally manipulated. In the analysis, we selected the subset of trials that included no repetitions of any kind, as in Experiment 1. To minimize the number of trials that included a repetition, we used a five-choice task with five stimulus colors and five locations (see Fig. 1).

\section{Method}

Participants Twenty-six undergraduates from the University of Iowa participated in the experiment. Two participants were eliminated for having error rates over $20 \%$. All had normal or corrected-to-normal vision and were right-handed.
Table 1 Results of four-way within-subjects ANOVA in Experiment 1. Cohen's $f$ was calculated from partial $\eta^{2} \mathrm{~s}$ reported from SPSS using G*Power 3.1.2

\begin{tabular}{llll}
\hline Effect & $F(1,17)$ & $p$ & Cohen's $f$ \\
\hline Previous (P.) Simon & 13.51 & $<.005$ & 0.89 \\
P flanker & 0.23 & .64 & 0.11 \\
Simon & 165.04 & $<.0001$ & 3.12 \\
Flanker & 75.49 & $<.0001$ & 2.10 \\
P. Simon $\times$ P. flanker & 0.56 & .46 & 0.18 \\
Simon $\times$ flanker & 1.55 & .23 & 0.30 \\
P. Simon $\times$ Simon & 16.72 & $<.001$ & 0.99 \\
P.flanker $\times$ flanker & 5.40 & $<.05$ & 0.56 \\
P. Simon $\times$ flanker & 1.83 & .19 & 0.32 \\
P. flanker $\times$ Simon & 1.15 & .30 & 0.26 \\
P. Simon $\times$ P.flanker $\times$ flanker & 2.03 & .17 & 0.34 \\
P. Simon $\times$ P. flanker $\times$ Simon & 0.13 & .72 & 0.09 \\
P. Simon $\times$ Simon $\times$ flanker & 0.55 & .47 & 0.17 \\
P. flanker $\times$ Simon $\times$ flanker & 2.94 & .10 & 0.41 \\
P. Simon $\times$ P. flanker $\times$ Simon $\times$ flanker & 0.81 & .38 & 0.21 \\
\hline
\end{tabular}



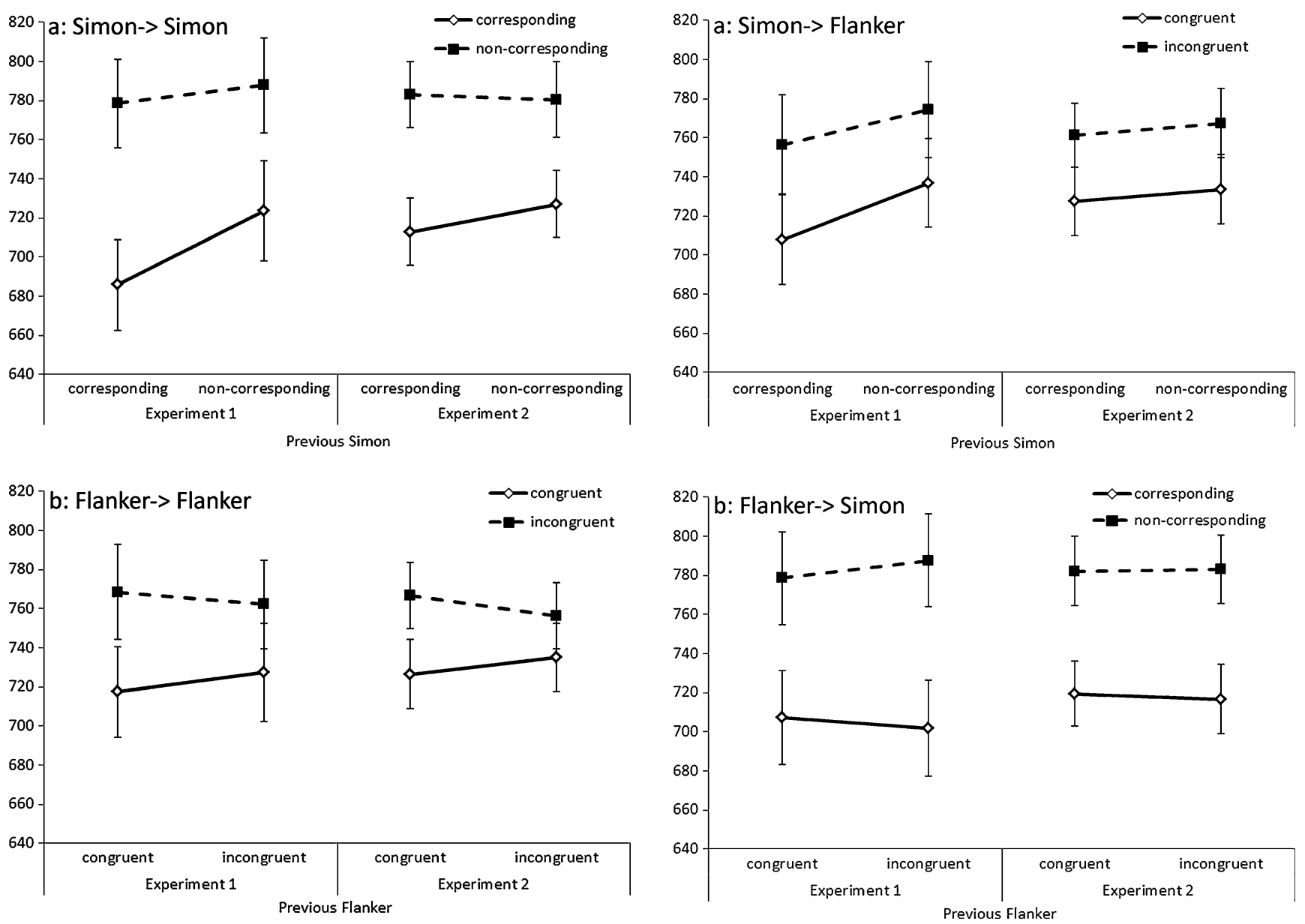

Fig. 2 Within-type conflict adaptation in a Simon conflict and b flanker conflict in both experiments. Error bars indicate standard errors of the means

Stimuli and apparatus Experiment 2 was identical to Experiment 1, except that (1) we used an additional color (violet, RGB value 216-25-255) to make the task a fivechoice task and a fifth stimulus location (Fig. 1b) and (2) participants responded with keys $<$ space $>-\mathrm{h}-\mathrm{j}-\mathrm{k}-\mathrm{l}$ on the keyboard, using their right hand (with the thumb on the space bar).

Design and procedure Experiment 2 was identical to Experiment 1, except that, as was mentioned above, we allowed repetitions of flankers and location, while not allowing response repetitions. For analysis, we again excluded all trials that involved any kind of repetition or negative priming.

\section{Results}

Because we excluded $50.6 \%$ of trials with some form of repetition from the analysis, we were not able to carry out an omnibus ANOVA in Experiment 2. Instead, we carried
Fig. 3 Across-type conflict adaptation from a Simon to flanker conflict and $\mathbf{b}$ flanker to Simon conflict in both experiments. Error bars indicate standard errors the means

out four separate two-way ANOVAs, each corresponding to the possible conflict adaptation effects within and across Simon and flanker conflict. We excluded another $23 \%$ of the remaining trials on the basis of the same criterion as that in Experiment 1 (overall error rate was $12.6 \%-8.4 \%$ incorrect responses and $4.2 \%$ slow responses, but we excluded all trials following these trials as well). In this way, there were at least 33 observations per participant in each cell of the analysis. ${ }^{1}$

There was a significant two-way interaction between Simon conflict on the previous trial and Simon conflict on the current trial, $F(1,23)=4.73, p<.05$, Cohen's $f=0.45$. The Simon effect decreased from $69 \mathrm{~ms}$ after Simon

\footnotetext{
${ }^{1}$ We also carried out a four-way ANOVA analogous to the one in Experiment 1 . The only interaction effects that were significant were again the two-way interaction within Simon conflict on current and previous trials and the two-way interaction within flanker conflict on current and previous trials, closely replicating the result from Experiment 1 . Some cells in this analysis, however, had exceedingly small number of trials $(<3)$.
} 
corresponding trials to $53 \mathrm{~ms}$ after Simon noncorresponding trials (Fig. 2a). Similarly, there was a significant twoway interaction between flanker conflict on the previous trial and flanker conflict on the current trial, $F(1,23)=$ $4.66, p<.05$, Cohen's $f=0.45$. The flanker effect decreased from $40 \mathrm{~ms}$ after flanker congruent trials to $21 \mathrm{~ms}$ after flanker incongruent trials (Fig. 2b). Thus, there was significant within-conflict adaptation in both Simon and flanker conflict. The main effect of Simon and flanker conflict in all four ANOVAs was also highly significant.

There was no significant two-way interaction between flanker conflict on the previous trial and Simon conflict on the current trial, $F(1,23)=0.22, p=.64$, Cohen's $f=0.09$. Similarly, there was no significant two-way interaction between Simon conflict on the previous trial and flanker conflict on the current trial, $F(1,23)=0.00, p=.99$, Cohen's $f=0.0009$. Thus, there is no evidence for conflict adaptation across the two types of conflict (Fig. 3). In sum, Experiment 2 replicates the finding of local conflict adaptations that was found in Experiment 1.

\section{General discussion}

We factorially crossed flanker and Simon conflict within a single task in two experiments, eliminating all feature repetitions, and obtained two principal results. First, in both experiments, we found evidence for conflict adaptation within both Simon and flanker conflict after eliminating all feature repetitions, consistent with previous studies reporting conflict adaptation in the absence of repetitions. Critically, in neither experiment did we find evidence of across-type conflict adaptation between Simon conflict and Flanker conflict.

Is cognitive control local? These results generally support the domain specificity view of control, which hypothesizes that control does not operate in a generic way but, rather, acts locally, specific to the type of conflict, even within a given task. As such, the results are consistent with previous studies (Egner et al., 2007; Funes et al., 2010; Wendt et al., 2006) suggesting that conflict adaptation is domain specific. However, these studies did not distinguish between repetition effects and control effects. The present findings demonstrate that control effects are domain specific and are present in the absence of any repetition effects.

Our results are consistent with the conclusion of a recent review by Egner (2008), who suggested that cognitive control is always domain specific. While we agree that, so far, evidence certainly favors the position that cognitive control is generally recruited domain specifically, we would like to caution against a sweeping conclusion. As Egner noted, although there has been a flurry of studies asking whether control is local, only a fraction of these actually controlled for effects of task structure, which previous research showed also influences deployment of control (Akçay \& Hazeltine, 2008; Egner, 2008). Thus, there is limited evidence regarding whether control is specific to conflict types, and not all combinations of conflict types have been examined with respect to domain specificity.

Furthermore, some studies have shown conflict adaptation across conflict types, even in the face of apparent task switching from a flanker to a Stroop task (Freitas, Bahar, Yang, \& Banai, 2007). Similarly, Notebaert and Verguts (2008) found conflict adaptation across two different types of conflict when the same relevant information was used in both types of trials, but not when different types of information was used (indeed, a reverse conflict adaptation was present in the latter condition). These results stand in contrast with the experiments reported here, which used identical relevant information across two types of conflict. It remains possible that certain control processes are more or less general in scope (i.e., transfer to more than single types of conflict) and/or are deployed flexibly depending on the task at hand. The latter possibility is supported by our finding that control effects can be task specific even within a domain (Akçay \& Hazeltine, 2008), suggesting that the scope of conflict adaptation can be flexible even within a domain (see also Hazeltine, Lightman, Schwab, \& Schumacher, 2011). In short, the scope of control seems to be flexible, and the rules governing how control is deployed need further research.

Implications for models of cognitive control Recently, the debate over alternative explanations of conflict adaptation (i.e., those that invoke repetition effects vs. those that invoke cognitive control) has inspired new models of conflict adaptation in which researchers have tried to bridge the gap between the control and feature repetition accounts (Blais, Robidoux, Risko, \& Besner, 2007; Verguts \& Notebaert, 2008). For example, Verguts and Notebaert implemented a Hebbian learning model that can account for conflict adaptation effects, which are typically observed even after removing all types of repetitions (as in the present experiments). This model also is able to account for local conflict adaptation effects across two tasks, since the learning algorithm updates connection weights depending on their activation on the last trial. In the model, the task layer of the task presented on the last trial is more active than that on the other task, and therefore, the learning algorithm has a larger effect on the most recent task.

While the model was explicitly designed to model local conflict adaptation, it does not distinguish between task specificity and domain specificity. Nonetheless, the model can likely be extended to account for domain-specific conflict adaptation as well. In the model, learning occurs on both relevant and irrelevant connections, strengthening the 
former further and weakening the latter after a noncorresponding trial. Assuming that there is a single layer of relevant input, this learning algorithm will create global conflict adaptation, since after any type of conflict, the relevant layer connections will be strengthened. Therefore, to account for domain specificity within a single task, the learning algorithm will need to work mostly on the connections of the irrelevant layer, weakening and strengthening them after noncorresponding and corresponding trials, respectively. In any case, the principle of local adjustment of connection weights based on their current activation level can indeed explain domain-specific conflict adaptation (Akçay \& Hazeltine, 2008).

In summary, we found in two experiments that conflict adaptation within a task, independently of feature repetitions, was local within two different kinds of conflict. This finding adds to the accumulating evidence that cognitive control is local within specific domains of information. This finding provides evidence against a single, unitary control process and also against control that is confined to the modulation of the influence of individual stimulus features. However, it strikes us as unlikely that there are separate control mechanisms for each potential source of conflict. Given that, in some cases, control is local even within a single type of information (e.g., Akçay \& Hazeltine, 2008) and, in other cases, global across different types of information (Freitas et al., 2007; condition 1 in Notebaert \& Verguts, 2008), it would seem that features and dimensions are controlled on the basis of their salience in relation to the particular task demands confronted by the system. How the boundaries of these control processes are determined remains an important question for those wishing to understand the implementation of control as it guides us through complex real-world environments.

Acknowledgements We would like to thank Rose Potter and Dustin Koth for running the participants and Serap Yiğit-Elliott, Wim Notebaert, Chris Blais, and an anonymous reviewer for their valuable comments on the manuscript.

\section{References}

Akçay, Ç., \& Hazeltine, E. (2007). Feature-overlap and conflict monitoring: Two sources of sequential modulations. Psychonomic Bulletin \& Review, 14, 742-748.

Akçay, Ç., \& Hazeltine, E. (2008). Conflict adaptation depends on task structure. Journal of Experimental Psychology: Human Perception and Performance, 34, 958-973.

Blais, C., Robidoux, S., Risko, E. F., \& Besner, D. (2007). Item specific adaptation and the conflict monitoring hypothesis: A computational model. Psychological Review, 114, 1076-1086.

Botvinick, M., Braver, T. S., Barch, D. M., Carter, C. S., \& Cohen, J. D. (2001). Conflict monitoring and cognitive control. Psychological Review, 108, 624-652.

Bugg, J. M. (2008). Opposing influences on conflict-adaptation in the Eriksen flanker task. Memory \& Cognition, 36, 1217-1227.
Egner, T. (2008). Multiple conflict-driven control mechanisms in the human brain. Trends in Cognitive Sciences, 12, 374-380.

Egner, T., Delano, M., \& Hirsch, J. (2007). Seperate conflict-specific cognitive control mechanisms in the human brain. Neuroimage, $35,940-948$.

Eriksen, B. A., \& Eriksen, C. W. (1974). Effects of noise letters upon the identification of a target letter in a nonsearch task. Perception \& Psychophysics, 16, 143-149.

Faul, F., Erdfelder, E., Lang, A.-G., \& Buchner, A. (2007). G*Power 3: A flexible statistical power analysis for the social, behavioral, and biomedical sciences. Behavior Research Methods, 39, 175-191.

Freitas, A. L., Bahar, M., Yang, S., \& Banai, R. (2007). Contextual adjustments in cognitive control across tasks. Psychological Science, 18, 1040-1043.

Funes, M. J., Lupiáñez, J., \& Humpreys, G. (2010). Analyzing the generality of conflict adaptation effects. Journal of Experimental Psychology: Human Perception and Performance, 36, 147-161.

Gratton, G., Coles, M. G. H., \& Donchin, E. (1992). Optimizing the use of information: Strategic control of activation of responses. Journal of Experimental Psychology: General, 121, 480-506.

Hazeltine, E., Lightman, E., Schwarb, H., \& Schumacher, E. H. (2011). The boundaries of sequential modulations: Evidence for set-level control. Manuscript under review

Hommel, B. (1997). Interactions between stimulus-stimulus congruence and stimulus-response compatibility. Psychological Research, 59, 248-260.

Hommel, B., Proctor, R. W., \& Vu, K. L. (2004). A feature-integration account of sequential effects in the Simon task. Psychological Research, 68, 1-17.

Kerns, J. G., Cohen, J. D., MacDonald, A. W., Cho, R. Y., Stenger, V. A., \& Carter, C. S. (2004). Anterior cingulate conflict monitoring and adjustments in control. Science, 303, 1023-1026.

Kiesel, A., Kunde, W., \& Hoffman, J. (2006). Evidence for taskspecific resolution of response conflict. Psychonomic Bulletin \& Review, 13, 800-806.

Kunde, W. (2003). Sequential modulations of stimulus-response correspondence effects depend on awareness of response conflict. Psychonomic Bulletin \& Review, 10, 198-205.

MacLeod, C. M. (1991). Half a century of research on the Stroop effect: An integrative review. Psychological Bulletin, 109, 163-203.

Mayr, U., Awh, E., \& Laurey, P. (2003). Conflict adaptation effects in the absence of executive control. Nature Neuroscience, 6, 450-452.

Monsell, S., \& Driver, J. (2000). Banishing the control homunculus. In S. Monsell \& J. Driver (Eds.), Control of cognitive processes: Attention and performance XVIII (pp. 3-32). Cambridge, MA: MIT Press.

Notebaert, W., \& Verguts, T. (2007). Dissociating conflict adaptation from feature integration: A multiple regression account. Journal of Experimental Psychology: Human Perception and Performance, $33,1256-1260$.

Notebaert, W., \& Verguts, T. (2008). Cognitive control acts locally. Cognition, 106, 1071-1080.

Simon, J. R., \& Rudell, A. P. (1967). Auditory S-R compatibility: The effect of an irrelevant cue on information processing. The Journal of Applied Psychology, 51, 300-304.

Stürmer, B., Leuthold, H., Soetens, E., Schröter, H., \& Sommer, W. (2002). Control over location-based response activation in the Simon task: Behavioral and electrophysiological evidence. Journal of Experimental Psychology: Human Perception and Performance, 28, 1345-1363.

Verguts, T., \& Notebaert, W. (2008). Hebbian learning of cognitive control: Dealing with specific and nonspecific adaptation. Psychological Review, 115, 518-525.

Wendt, M., Kluwe, R. H., \& Peters, A. (2006). Sequential modulations of interference evoked by processing task-irrelevant stimulus features. Journal of Experimental Psychology: Human Perception and Performance, 32, 644-667. 